\title{
Unstable particles in finite volume: The broken phase of the 4-d $O(4)$ non-linear $\sigma$-model
}

\author{
Frank Zimmermann ${ }^{\mathrm{a}}$ *, Jörg Westphalen ${ }^{\mathrm{a}}$, \\ Meinulf Göckeler ${ }^{\mathrm{a}, \mathrm{b}, \dagger}$ and Hans A. Kastrup ${ }^{\mathrm{a}}$ \\ a Institut für Theoretische Physik E, RWTH Aachen, W-5100 Aachen, FRG \\ b HLRZ c/o KFA Jülich, P.O.Box 1913, W-5170 Jülich, FRG
}

According to a proposal of Lüscher it is possible to determine elastic scattering phases in infinite volume from the energy spectrum of two-particle states in a periodic box. We demonstrate the applicability of this method in the broken phase of the 4-dimensional $O(4)$ non-linear $\sigma$-model in a Monte-Carlo study on finite lattices. This non-perturbative approach also permits the study of unstable particles, the $\sigma$-particle in our case. We observe the $\sigma$-resonance and extract its mass and its width.

\section{INTRODUCTION}

Recently Lüscher derived a relation between the energy spectrum of two-particle states in a finite box with periodic boundary conditions and elastic scattering phase shifts defined in infinite volume [1]. Since two-particle energy levels are calculable by Monte Carlo techniques, this relation opens the possibility to extract phase shifts from numerical simulations on finite lattices. The scattering lengths have already been examined by means of the asymptotic large-volume behaviour of the two-particle energy levels [2]. Furthermore, Lüscher's relation for two dimensions has recently been used to extract the scattering phase shifts of the $O(3)$ non-linear $\sigma$-model [3] and to study reso-

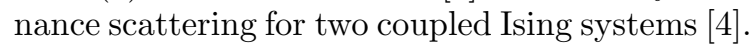
As a next step towards the determination of resonance parameters in QCD [5] we show the applicability of the relation in the 4-dimensional $O(4)$ non-linear $\sigma$-model. We investigate this model in its broken phase, where the $\sigma$-particle is unstable.

In order to study two-particle scattering in finite (spatial) volume Lüscher starts with a nonrelativistic model considering two identical massive bosons in a periodic cubic box. The box size is assumed to be larger than the interaction range. He shows that the energy spectrum is determined

${ }^{*}$ speaker at the conference

${ }^{\dagger}$ supported by the Deutsche Forschungsgemeinschaft by the scattering phases at the energy eigenvalues. For states below the inelastic threshold this result is then carried over to massive relativistic quantum field theories by invoking an effective Schrödinger equation.

Since this whole reasoning only works in the absence of massless (physical) particles, we have to equip the Goldstone bosons with a mass before we can apply Lüscher's formulas. So we add an external source term to the lattice action of the 4-dimensional $O(4)$ non-linear $\sigma$-model:

$S=-2 \kappa \sum_{x} \sum_{\mu=1}^{4} \Phi_{x}^{\alpha} \Phi_{x+\hat{\mu}}^{\alpha}+J \sum_{x} \Phi_{x}^{4}$.

The scalar field is represented as a four component vector $\Phi_{x}^{\alpha}$ of unit length: $\Phi_{x}^{\alpha} \Phi_{x}^{\alpha}=1$.

\section{THEORETICAL BACKGROUND}

Consider a system of two Goldstone bosons ("pions") of mass $m_{\pi}$ with zero total momentum in a cubic box of size $L^{3}$. The two-particle states in the elastic region are characterised by centre-ofmass energies $W$ or momenta $\vec{k}$ defined through

$W=2 \sqrt{m_{\pi}^{2}+\vec{k}^{2}}, \quad k=|\vec{k}|$,

with $W$ and $k$ in the ranges:

$2 m_{\pi}<W<4 m_{\pi} \quad \Leftrightarrow \quad 0<k / m_{\pi}<\sqrt{3}$. 
They are classified according to irreducible representations of the cubic group. Their discrete energy spectrum $W_{j}, j=0,1,2, \ldots$, is related to the scattering phase shifts $\delta_{l}$ with angular momenta $l$ which are allowed by the cubic symmetry of the states [1].

In the subspace of cubically invariant states only angular momenta $l=0,4,6, \ldots$ contribute. Due to the short-range interaction it seems reasonable to neglect all $l \neq 0$. Then an energy value $W_{j}$ belongs to the two-particle spectrum, if the corresponding momentum $k_{j}=\sqrt{\left(W_{j} / 2\right)^{2}-m_{\pi}^{2}}$ is a solution of

$\delta_{0}\left(k_{j}\right)=-\phi\left(\frac{k_{j} L}{2 \pi}\right)+j \pi$.

The continuous function $\phi(q)$ is given by

$\tan (-\phi(q))=\frac{q \pi^{3 / 2}}{\mathcal{Z}_{00}\left(1, q^{2}\right)} \quad, \quad \phi(0)=0$,

with $\mathcal{Z}_{00}\left(1, q^{2}\right)$ defined by analytic continuation of

$\mathcal{Z}_{00}\left(s, q^{2}\right)=\frac{1}{\sqrt{4 \pi}} \sum_{\vec{n} \in \mathbf{Z}^{3}}\left(\vec{n}^{2}-q^{2}\right)^{-s}$.

This result holds only for $0<q^{2}<9$, which is fulfilled in our simulations, and provided finite volume polarisation effects and scaling violations are negligible [1].

In our Monte-Carlo investigation we calculate the momenta $k_{j}$ corresponding to the two-particle energy spectrum $W_{j}$ for a given size $L$, and can then read off the scattering phase $\delta_{0}\left(k_{j}\right)$ from eq. (何. In order to scan the momentum dependence of the phase shift we have to vary the spatial extent $L$ of the lattice.

\section{HOW TO EXTRACT THE TWO- PARTICLE SPECTRUM}

The two-particle states in our model are also classified according to the remaining $O(3)$-symmetry: They have "isospin" $0,1,2$. Since we expect the $\sigma$-resonance to be in the isospin- 0 channel, we restrict ourselves to that case. The corresponding two-particle operators on a lattice of spatial ex- tent $L$ are defined according to

$\mathcal{O}_{i}(t)=\sum_{a=1}^{3} \sum_{\vec{p}} f_{i}(\vec{p}) \tilde{\Phi}_{-\vec{p}, t}^{a} \tilde{\Phi}_{\vec{p}, t}^{a}, \quad \vec{p} \in \mathbf{Z}_{\mathbf{L}}^{3}$

where $f_{i}(\vec{p}), i=1,2, \ldots$ is some basis of wave functions and $\tilde{\Phi}_{\vec{p}, t}^{\alpha}$ is the spatial Fourier transform of the lattice field $\Phi_{x}^{\alpha}$ :

$\tilde{\Phi}_{\vec{p}, t}^{\alpha}=L^{-3} \sum_{\vec{x}} \Phi_{\vec{x}, t}^{\alpha} e^{2 \pi i \vec{x} \cdot \vec{p} / L}$.

We want to compare two different kinds of cubically invariant wave functions. First we take plane waves,

$f_{i}(\vec{p})=\delta_{i, \vec{p}^{2}} \quad, \quad i=1,2, \ldots$

Our second choice is motivated by the periodic singular solutions of the Helmholtz equation (see (11),

$f_{i}(\vec{p})=\left(\vec{p}^{2}-q_{i}^{2}\right)^{-1} \quad, \quad i=1,2, \ldots$,

with values $q_{i}=(L / 2 \pi) \sqrt{\left(W_{i} / 2\right)^{2}-m_{\pi}^{2}}$ using as input the plane wave energy spectrum $W_{i}$ of some test runs. These latter wave functions should have an enhanced projection especially at energies close to the resonance mass.

We also take into account the $\sigma$-field at zero momentum, since it has the same quantum numbers as $\mathcal{O}_{i}(t)$ defined in eq. (7):

$\mathcal{O}_{0}(t)=\tilde{\Phi}_{\overrightarrow{0}, t}^{4}=L^{-3} \sum_{\vec{x}} \Phi_{\vec{x}, t}^{4}$.

The eigenvalues of the two-particle correlation function matrix

$C_{i j}(t)=\left\langle\left(\mathcal{O}_{i}(t)-\mathcal{O}_{i}(t+1)\right) \mathcal{O}_{j}(0)\right\rangle$

with $i, j=0,1,2, \ldots$ should decay proportional to $\exp \left(-W_{i} t\right)$. Up to exponentially suppressed corrections this also holds if the set of states is truncated at some value $i=r$ such that $W_{r}$ is above the inelastic threshold. In this way we get a system of independent states inside the elastic region [3]. In our simulations this requirement is fulfilled for $r \leq 6$ depending on $\kappa$ and $L$. 


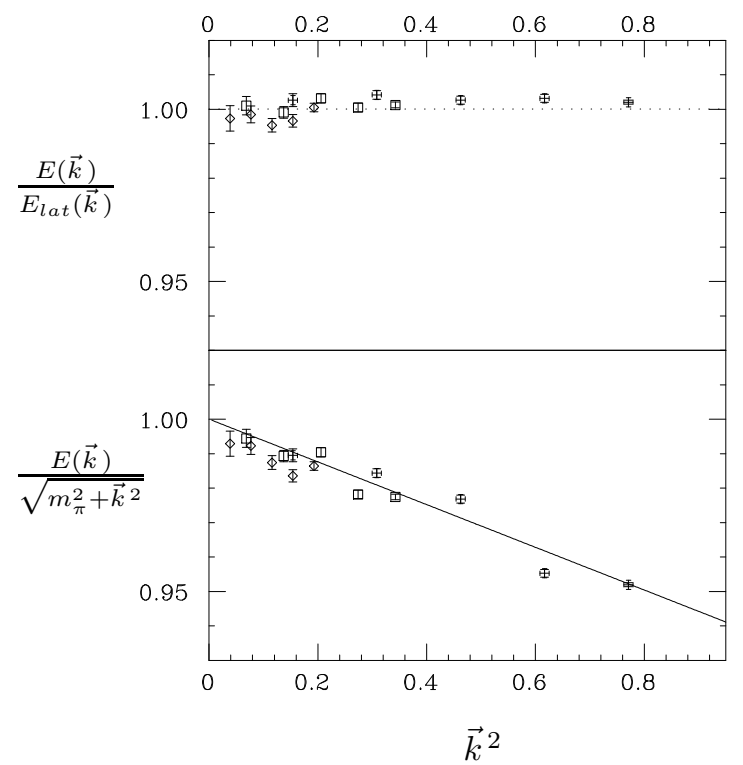

Figure 1. Single particle energy values $E(\vec{k}) d i$ vided by the lattice and the continuum dispersion relation, respectively, as function of $\vec{k}^{2}$

\section{TECHNICAL DETAILS}

In order to generate our configurations we used the reflection cluster algorithm in its multi-cluster version generalised to the case of finite external source $J$. The Fourier transform $\tilde{\Phi}_{\vec{p}, t}$ is computed by a 3-dimensional FFT. In the case of the plane waves we also used the 2-cluster method [3] to check our results for the correlation matrix (12). The hopping parameter $\kappa=0.315$ and the external source $J=0.01$ are tuned such that the $\sigma$-mass lies in the elastic region.

The simulations are performed on cylindrical lattices $L^{3} \cdot T$. On the HLRZ-CRAY Y-MP8/832 we can only run lattices of sizes $16^{3} \cdot 32$ and $20^{3} \cdot 32$. The larger lattices $24^{3} \cdot 32,32^{3} \cdot 40$ are simulated on the "Landesvektorrechner" SNI S600/20 in Aachen. In order to check the dependence on $T$ we have performed runs on a lattice $24^{3} \cdot 60$, but without getting significant changes of the results.

Instead of calculating the eigenvalues of $C(t)$ we follow refs. [3, 4], choose some small $t_{0}$ and diagonalise $C^{-1 / 2}\left(t_{0}\right) C(t) C^{-1 / 2}\left(t_{0}\right)$. After blocking the data we determine the mean values and errors using the jackknife method. Finally the energy values are extracted by a 1-parameter fit to the mean eigenvalues according to the ansatz: $\exp \left(-\left(t-t_{0}\right) W_{i}\right)$.

Variations of the rank $(r+1)$ of the correlation function matrix have only little influence on the results, while $t_{0}$ must be chosen small in order to guarantee numerical stability of the eigenvalue calculation.

Furthermore we have developed a method to determine the upper end $t_{\max }$ of the fit range dynamically. The eigenvalues are sorted such that the corresponding eigenvectors at successive time slices are as parallel as possible. This should be equivalent to a sorting with respect to the size of the eigenvalues, but due to statistical fluctuations at some time slice $t=t_{\max }+1$ this fails, thus determining $t_{\max }$.

\section{NUMERICAL RESULTS}

In order to control scaling violations, we first examine the single particle energies extracted from the exponential decay of the propagator

$$
\left\langle\sum_{a=1}^{3} \sum_{\vec{p}} \delta_{i, \vec{p}^{2}} \tilde{\Phi}_{-\vec{p}, t}^{a} \tilde{\Phi}_{\vec{p}, 0}^{a}\right\rangle \sim e^{-E(\vec{k}) t}
$$

with $\vec{k}^{2}=(2 \pi / L)^{2} i$. Fig. 11 shows that the data are well approximated either by

$E(\vec{k}) \approx \sqrt{m_{\pi}^{2}+\vec{k}^{2}}\left(1-0.06(1) \vec{k}^{2}\right)$

or by the lattice dispersion relation $\left(\hat{k}_{\nu}=2 \sin \frac{k_{\nu}}{2}\right)$ :

$E(\vec{k}) \approx E_{\text {lat }}(\vec{k})=2 \operatorname{asinh}\left(\frac{1}{2} \sqrt{m_{\pi}^{2}+\overrightarrow{\hat{k}}^{2}}\right)$.

Under the assumption that polarisation effects are exponentially suppressed by a factor of $\exp \left(-m_{\pi} L\right)$ we get for the lowest state $(\vec{k}=\overrightarrow{0})$ the infinite volume mass $m_{\pi}=0.229(1)$.

Fig. 2 2 shows our preliminary results for the twoparticle energy spectrum. The points connected by solid lines are the numerical results, bounded from above by the inelastic threshold $4 m_{\pi}$. We get about the same numbers for both kinds of wave functions (9), (10). The lowest value represents the bound ground state, which is not relevant for the discussion of scattering states. The 


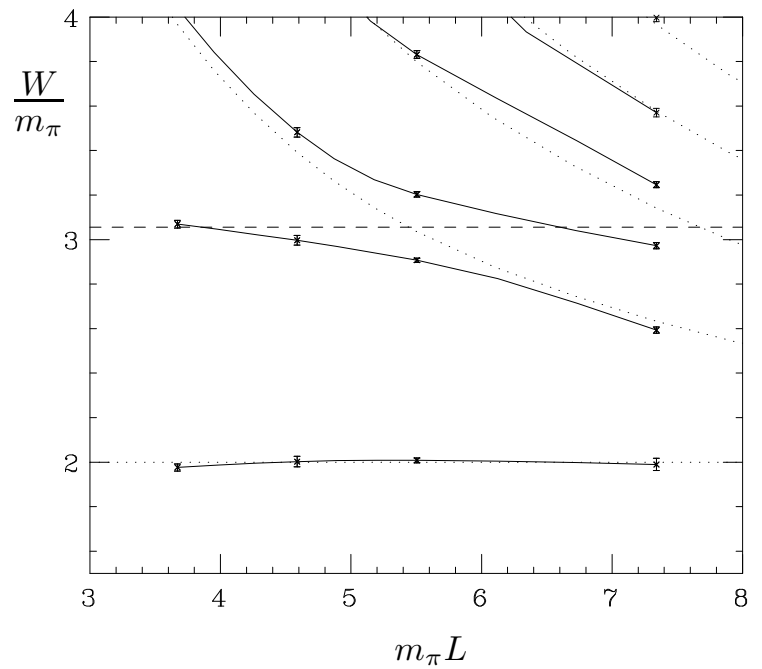

Figure 2. Two-particle energy spectrum

dotted lines correspond to the free energy spectrum:

$$
\frac{W}{m_{\pi}}=2 \sqrt{1+\frac{\vec{k}^{2}}{m_{\pi}}}, \quad \vec{k}=\frac{2 \pi}{L} \vec{n}, \quad \vec{n} \in \mathbf{Z}^{3},
$$

with $\vec{n}^{2}=0,1,2, \ldots$ We see the expected trend: the first and second level are coming close to each other forming the so called "avoided level crossing", such that we get an impression where the resonance plateau (dashed line) might be.

For each lattice extent $L$ and energy level $W_{i}$ in the elastic region the corresponding scattering phase shift is calculated according to eq. (4). Fig. 3 summarises the results. The vertical dotted line represents the inelastic threshold (see eq. (3)).

Finally we perform a fit to the data according to the Breit-Wigner resonance formula (solid line)

$\tan \left(\delta-\frac{\pi}{2}\right)=\frac{W-m_{\sigma}}{\Gamma_{\sigma} / 2}$

determining the mass and the width of the $\sigma$ resonance:

$m_{\sigma}=3.07(1) m_{\pi} \quad, \quad \Gamma_{\sigma}=0.19(2) m_{\pi}$.

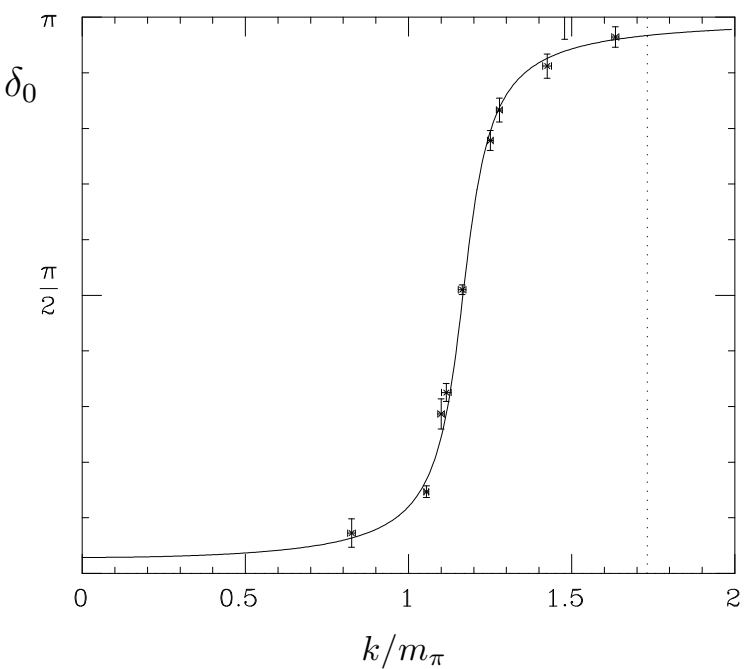

Figure 3. Scattering phase shift $\delta_{0}$ in the isospin 0 channel

\section{DISCUSSION AND CONCLUSIONS}

The resonance mass $m_{\sigma}$ is about the same as the mass we have obtained from a propagator fit. Because of triviality, perturbation theory should be applicable in this model, but the tree level approximation gives about three times the numerical value for $\Gamma_{\sigma}$ in eq. (18). This deviation could at least partially be caused by lattice effects. For further details the reader is referred to $[6]$.

\section{ACKNOWLEDGEMENT}

Helpful discussions with C. Frick and M. Lüscher are gratefully acknowledged. Special thanks are due to T. Neuhaus for allowing us to use his programs as basis of our programming. Furthermore we wish to thank the Rechenzentrum at the RWTH Aachen and the HLRZ Jülich for providing the necessary computer time.

\section{REFERENCES}

1. M. Lüscher, Nucl. Phys. B354 (1991) 531.

2. C. Frick, K. Jansen, J. Jersák, I. Montvay, G. Münster and P. Seuferling, Nucl. Phys. B331 (1990) 515; J. Nishimura, Tokyo preprint UT-615-92. 
3. M. Lüscher and U. Wolff, Nucl. Phys. B339 (1990) 222.

4. C.R. Gattringer and C.B. Lang, Phys. Lett. B274 (1992) 95, preprint UNIGRAZ-UTP-0405-92, talk at this conference.

5. M. Lüscher, Nucl. Phys. B364 (1991) 237.

6. M. Göckeler, H.A. Kastrup, J. Westphalen, F. Zimmermann, in preparation. 